VOL. 44 (1991) [253-261]

\title{
THE EXTREME POINTS OF A CLASS OF FUNCTIONS WITH POSITIVE REAL PART
}

\author{
N. SAMARIS
}

Let $P_{1}$ be the class of holomorphic functions on the unit disc $U=\{z:|z|<1\}$ for which $f(0)=1$ and $\operatorname{Re} f>0$. Let also $P_{n}$ be the corresponding class on the unit $\operatorname{disc} U^{n}$. The inequality $\left|a_{k}\right| \leqslant 2$ is known for the Taylor coefficients in the class $P_{1}$. In this paper, it is generalised for the class $P_{n}$. If $\rho=\left(\rho_{1}, \rho_{2}, \ldots, \rho_{n}\right)$, with $\rho_{1}, \rho_{2}, \ldots, \rho_{n}$ nonegative integers whose greatest common divisor is equal to 1 , we describe the form of the functions $f \in P_{n}$ under the restriction $\left|a_{\rho}\right|=2$. Under the same restriction, we give conditions for a function to be an extreme point of the class $P_{n}$.

\section{INTRODUCTION}

Let $U$ be the open unit disc in the complex plane $C$. If $n$ is any natural number, $P_{n}$ represents the class of all holomorphic functions in $U^{n}$, which have positive real part and assume the value 1 at the origin $\theta=(0,0,0, \ldots, 0)$. These functions can be expanded in Taylor series of the form

$$
f(z)=\sum_{k \geqslant 0} a_{k} z^{k}
$$

where: $k=\left(k_{1}, k_{2}, \ldots, k_{n}\right) \in \mathbb{N}_{0}^{n}, \mathbb{N}_{0}=\{0,1,2, \ldots\}, z^{k}=z_{1}^{k_{1}} z_{2}^{k_{2}} \ldots z_{n}^{k_{n}}, a_{\theta}=1$ and $\operatorname{Re} f>0$.

In the case of $n=1$, the Carathéodory-Toeplitz determinants describe the behaviour of the Taylor coefficients of class $P_{1}$. An immediate conclusion is the Carathéodory relation $\left|a_{k}\right| \leqslant 2, k=1,2, \ldots$ and that if $a_{1}=2 e^{i \varphi}$, then $a_{k}=2 e^{i k \varphi}$ and $f(z)=\left(1+e^{i \varphi} z\right)\left(1-e^{i \varphi} z\right)^{-1}$. Moreover the functions of the above type constitute the extreme elements of the class $P_{1}$ (see [3]).

References $[1,2]$ deal with the problem of locating the extreme elements of the class $P_{2}$ and some of them were located.

In Section 2, by Theorem 2.3 of the present study, we achieve a generalisation of Carathéodory's conclusion for any class $P_{n}$ in the case of $a_{p}=2 e^{i \varphi}$, where $\rho=$ $\left(\rho_{1}, \rho_{2}, \ldots, \rho_{n}\right) \geqslant \theta$ and $\rho_{1}, \rho_{2}, \ldots, \rho_{n}$ are numbers prime to each other.

Received 12 October 1990

Copyright Clearance Centre, Inc. Serial-fee code: 0004-9729/91 \$A2.00+0.00. 
The above are related to Theorem 3.

For the deduction of the results in Section 2 some simple relations from classical harmonic analysis will be used.

In Section 3, we will investigate the problem of locating the extreme points of any class $P_{n}$, by means of Theorem 2.3 , and it will become possible to find some of them.

\section{SOME PROPERTIES OF CLASS $\boldsymbol{P}_{\boldsymbol{n}}$}

LEMMA 2.1. If $K(x)=K\left(x_{1}, x_{2}, \ldots x_{n}\right)$ is a Lebesgue integrable function in $\mathbb{R}^{n}$, such that $\operatorname{Re} K \geqslant 0$, and $\widehat{K}(t)=\int_{\mathbb{R}^{n}} e^{-i x t} K(x) d x$ is the Fourier transform of $K(x)$, then the relation

$$
|\widehat{K}(t)+\overline{\widehat{K}}(-t)| \leqslant 2 \operatorname{Re} \widehat{K}(0)
$$

holds for all $t \in \mathbb{R}^{n}$.

The proof is straighi-forward by the definition of the Fourier transform.

LEMMA 2.2. If $K(x)$ is a function as in Lemma 2.1, such that the function $\widehat{K}$ has compact support and $K \geqslant 0$, then for every $f(z)=\sum_{\rho \geqslant \theta} a_{\rho} z^{\rho} \in P_{n}$ the inequaltiy $\left|\sum_{\rho \geqslant \theta} a_{\rho} \widehat{K}(s-\rho)+\sum_{\rho \geqslant \theta} \bar{a}_{\rho} \overline{\widehat{K}}(-s-\rho)\right| \leqslant 2 \operatorname{Re} \sum_{\rho \geqslant \theta} a_{\rho} \widehat{K}(-\rho)$ holds for every $s \in \mathbb{R}^{n}$.

PROOF: If $z=r e^{i x}=\left(r_{1} e^{i x_{1}}, r_{2} e^{i x_{2}}, \ldots, r_{n} e^{i x_{n}}\right)$ and $F_{r}(x)=K(x) f\left(r e^{i x}\right)=$ $\left(\sum_{\rho \geqslant \theta} a_{\rho} r^{\rho} . e^{i \rho x}\right) K(x)$ then the function $F_{r}(x)$ satisfies the conditions of Lemma 2.1 . We apply this Lemma, take the limit as $r \rightarrow(1,1, \ldots, 1)$ and the requested result is obtained.

THEOREM 2.3. If $f(z)=\sum_{k \geqslant \theta} a_{k} z^{k} \in P_{n}$, then:

(a) $\left|a_{k}\right| \leqslant 2, k \in \mathbb{N}_{0}^{n}$

(b) If a certain index $\rho=\left(\rho_{1}, \rho_{2}, \ldots \rho_{n}\right)$ with $\rho_{1}, \rho_{2}, \ldots, \rho_{n}$ numbers prime to each other, satisfies the relation $a_{\rho}=2 e^{i \varphi}$ then:

(i) $a_{\lambda \rho}=2 e^{i \lambda \varphi}$, for every natural number $\lambda$.

(ii) If $\theta \leqslant k \leqslant \rho, k \neq \rho, k \neq \theta$ then $a_{k}=\bar{a}_{\rho-k} e^{i \varphi}$ and $a_{k+\lambda \rho}=$ $a_{k} e^{i \lambda \varphi}$, for every natural number $\lambda$.

(iii) If for some index $k$ none of the relations $k \geqslant \rho, k \leqslant \rho$ is valid then $a_{k}=a_{k+\lambda \rho}=0$, for every natural number $\lambda$.

ProOF: (a) If we set $K(x)=\prod_{k=1}^{n}\left(\sin ^{2} \delta_{k} x_{k}\right) / x_{k}^{2}$, we obtain $\widehat{K}(t)=1 / \pi^{n} \prod \sup \left(0,2 \delta_{k}-\left|t_{k}\right|\right)$. By setting $\delta_{1}=\delta_{2}=\cdots=\delta_{n}=1 / 2$ and applying Lemma 2.2 we have the requested result. 
(b) Let $A$ be a rotation transform of $\mathbb{R}^{n}$, such that $A \rho=(|\rho|, 0,0 \ldots, 0)$. Since the inverse matrix of $A$ is equal to its transpose, we have the relation $t .\left(A^{-1} x\right)=$ $(A t) x$. If we set $P(x)=K(A x)$ and $x^{\prime}=A x$ we obtain:

$$
\begin{gathered}
\widehat{P}(t)=\int_{\mathbb{R}^{n}} K(A x) e^{i x t} d x=\int_{\mathbb{B}^{n}} K\left(x^{\prime}\right) e^{i t\left(A^{-1} x^{\prime}\right)} d x^{\prime}=\widehat{K}(A t) \text { or } \\
\widehat{P}\left(A^{-1} t\right)=\widehat{K}(t)=\frac{1}{\pi^{n}} \prod_{k=1}^{n} \sup \left(0,2 \delta_{k}-\left|t_{k}\right|\right)
\end{gathered}
$$

Since the matrix $A^{-1}$ leaves lengths and angles invariant, the rectangular region $S=\prod_{k=1}^{n}\left(-2 \delta_{k}, 2 \delta_{k}\right)$ is transformed into $A^{-1}(S)$ which has the same dimensions as $S$.

If we set $\delta_{1}=|\rho|$, then for suitably small $\delta_{2}, \delta_{3}, \ldots \delta_{n}$ no integer indices are contained in $A^{-1}(S)$ other than $\theta, \rho,-\rho$. This is further supported by the fact that the numbers $\rho_{1}, \rho_{2}, \ldots, \rho_{n}$ are prime to each other, so that the line segment $(-2 \rho, 2 \rho)$ contains no integer indices other than $\theta, \rho,-\rho$. In the above mentioned case we have:

$$
\begin{aligned}
\widehat{P}(\theta)=(2 / \pi)^{n} \delta_{1} \delta_{2} \ldots \delta_{n}, \widehat{P}(\rho) & =\widehat{K}(|\rho|, 00 \ldots 0) \\
& =\left(2^{n-1} / \pi^{n}\right) \delta_{1} \delta_{2} \ldots \delta_{n}=\widehat{P}(-\rho)
\end{aligned}
$$

and $\widehat{P}(k)=0$ for all the rest of the indices. Now applying Lemma 2.2 we obtain the relation:

$$
\left|a_{k-\rho}+a_{k+\rho}+2 a_{k}+\bar{a}_{\rho-k}\right| \leqslant 2 \operatorname{Re}\left(2+a_{\rho}\right) \text { for every } k \in \mathbb{N}_{0}^{n}
$$

(it is understood that $a_{s}=0$ when $s \neq \theta$ ).

If part (b) holds for the case $a_{\rho}=-2$ it holds generally. Indeed, if we consider the function $g(z)=f\left(\eta_{1} . z_{1}, z_{2}, \ldots, z_{n}\right)$ where $a_{\rho}=2 \eta$ and $\eta=-\eta_{1}^{-\rho_{1}}(|\eta|=1)$, we observe that the Taylor coefficient of order $\rho$ of the function $g$ is equal to -2 .

Applying now (b) in this case, we obtain the required result for the function $f$.

The hypothesis $a_{\rho}=-2$ yields the relations:

$$
\begin{aligned}
& a_{k-\rho}+a_{k+\rho}+2 a_{k}+\bar{a}_{-k-\rho}=0 \\
& a_{k}+a_{k+2 \rho}+a_{k+\rho}=0 \\
& a_{k+\lambda \rho}+a_{k+(\lambda+2) \rho}+a_{k+(\lambda+1) \rho}=0 \quad(\lambda+1)
\end{aligned}
$$

Subtracting successively the above relations we have:

$$
\omega=\left(a_{k+2 \rho}-a_{k}\right)=-\left(a_{k+3 \rho}-a_{k+\rho}\right)=+\left(a_{k+4 \rho}-a_{k+2 \rho}\right)=\ldots
$$


and consequently:

$$
a_{k+2 \lambda \rho}=a_{k}+\lambda \omega, \quad a_{k+\rho}+\lambda \omega=a_{k+(2 \lambda+1) \rho}
$$

Since $\left|a_{s}\right| \leqslant 2$ for every index $s$ we infer that $\omega=0$, so that returning to equations (0) and (1) in the case of $k \leqslant \rho, k \neq \theta, k \neq \rho$ we obtain that $a_{k}=-\bar{a}_{\rho-k}$; furthermore, if $k \ngtr \rho, k \neq \rho, a_{k}=0$ and for any $k \neq \theta$ it is true that $a_{k+\lambda \rho}=(-1)^{\lambda} a_{k}$.

\section{Extreme elements of Class $P_{n}$}

Let $S \subset N_{0}^{n}$ such that $\theta \in S . H S$ represents the set of the holomorphic functions on $U^{n}$ which assume the form:

$$
f(z)=\sum_{n \in S} a_{n} z^{n}, \quad \text { with } a_{\theta}=1
$$

By $P S$ we denote the set $P_{n} \cap H S$. Let $S_{\rho} \subset N_{0}^{n}$ for which the following conditions hold:

( $\alpha) \quad \rho=\left(\rho_{1}, \rho_{2}, \ldots, \rho_{n}\right) \in N_{0}^{n}$ with $\rho_{1}, \rho_{2}, \ldots, \rho_{n}$ numbers prime to each other;

( $\beta) \quad n \leqslant \rho$ and $n \neq \rho$ for every $n \in S_{\rho}$

( $\gamma) \quad \theta \in S_{\rho}$;

( $\delta$ ) when $n \leqslant \rho$ and $n \neq \theta$ then exactly one of the indices $n, \rho-n$ belongs to $S_{\rho}$.

If $f \in H N_{0}^{n}$, which satisfies the propositions (i), (ii), (iii) of Theorem 2.3 for $\varphi=0$, then, obviously,

$$
f(z)=\left[p(z)+z^{\rho} \bar{p}\left(\frac{1}{\bar{z}}\right)\right]\left(1-z^{\rho}\right)^{-1} \text { and } p \in H S_{\rho} .
$$

The inverse is also obvious.

If $\Omega_{p}(\varphi)=\left\{\sum_{n \geqslant \theta} a_{n} z^{n} \in P_{n}: a_{\rho}=2 e^{i \varphi}\right\}$ then on the above basis we are able to define the class $Q S_{\rho}$ by means of the relation

$$
\Omega_{\rho}(0)=\left\{\left[p(z)+z^{\rho} \bar{p}\left(\frac{1}{\bar{z}}\right)\right]\left(1-z^{\rho}\right)^{-1}: p \in Q S_{\rho}\right\} .
$$

If $E A$ represents the set of the extreme points of the convex set $A$, it is evident that

(i) $\Omega_{\rho}(0) \cap E P_{n}=\left\{\left[p(z)+z^{\rho} \bar{p}(1 / \bar{z})\right]\left(1-z^{\rho}\right)^{-1}: p \in E Q S_{\rho}\right\}$;

(ii) $\Omega_{p}(\varphi) \cap E P_{n}=\left\{f\left(e^{i \varphi} z_{1}, z_{2}, \ldots, z_{n}\right): f \in \Omega_{p}(0) \cap E P_{n}\right\}$. 
The following theorem provides a useful necessary and sufficient condition for a function to belong to $Q S_{\rho}$.

TheOREM 3.1. If $p \in H S_{\rho}, \rho^{*}=\left(\rho_{2}, \rho_{3}, \ldots, \rho_{n}\right)$ and $\tilde{\rho}=\rho_{1}+\rho_{2}+\cdots+\rho_{n}$ the following are equivalent:

(i) $p \in Q S_{\rho}$;

(ii) $\operatorname{Re} p\left(z^{-\rho^{*}}, z^{-\rho^{*}} z_{2}^{\tilde{\rho}}, \ldots, z^{-\rho^{*}} z_{n}^{\tilde{\rho}}\right) \geqslant 0$ for every $z=\left(z_{2}, z_{3}, \ldots, z_{n}\right) \in$ $(\partial U)^{n-1}$.

Proof: (i) $\rightarrow$ (ii).

If $f(z)=\left[p(z)+z^{\rho} \bar{p}(1 / \bar{z})\right]\left(1-z^{\rho}\right)^{-1}, p \in H S_{\rho}$ and $s=\left(s_{2}, s_{3}, \ldots, s_{n}\right) \in$ $(\partial U)^{n-1}$ then

$$
f\left(z_{1}, s_{2} z_{1}, \ldots, s_{n} z_{1}\right)=\sum_{k=0}^{\tilde{\rho}-1} \lambda_{k}(s)\left(\varepsilon_{k}+z_{1}\right)\left(\varepsilon_{k}-z_{1}\right)^{-1}
$$

where $\left\{\varepsilon_{k}\right\}_{k=0}^{\tilde{\rho}-1}$ are the solutions of the equation

$$
z_{1}^{\rho_{1}} . s_{2}^{\rho_{2}} \ldots s_{n}^{\rho_{n}}=1 \text { and } \lambda_{k}(s)=\operatorname{Re} p\left(\varepsilon_{k}, s_{2} \varepsilon_{k}, \ldots, s_{n} \varepsilon_{k}\right) \tilde{\rho}^{-1} .
$$

Indeed, it is obvious that

$$
f\left(z_{1}, s_{1} z_{1}, \ldots, s_{n} z_{n}\right)=\sum_{k=0}^{\tilde{\rho}-1} \lambda_{k}(s)\left(\varepsilon_{k}+z_{1}\right)\left(\varepsilon_{k}-z_{1}\right)^{-1}+C(s)
$$

where

$$
\begin{gathered}
\lambda_{k}(s)=\lim _{z_{1} \rightarrow e_{k}} f\left(z_{1}, s_{2} z_{1}, \ldots, s_{n} z_{1}\right) \cdot\left(\varepsilon_{k}-z_{1}\right)\left(\varepsilon_{k}+z_{1}\right)^{-1} \\
=\tilde{\rho}^{-1} \operatorname{Re} p\left(\varepsilon_{k}, s_{2} \varepsilon_{k}, \ldots, s_{n} \varepsilon_{k}\right) .
\end{gathered}
$$

Because $\sum_{k=0}^{\tilde{\rho}-1} \tilde{\rho}^{-1} \operatorname{Re} p\left(\varepsilon_{k}, s_{2} \varepsilon_{k}, \ldots, s_{n} \varepsilon_{k}\right)=1$ we have that $C(s)=0$. If $f \in \Omega_{\rho}(0)$ and $f\left(z_{1}, s_{2} z_{1}, \ldots, s_{n} z_{1}\right)=1+\sum_{n=1}^{\infty} \beta_{n}(s) z_{1}^{n}$

$$
\bar{\beta}_{\tilde{\rho}-n}(s)=2 \sum_{k=0}^{\tilde{\rho}-1} \lambda_{k}(s) \bar{\varepsilon}_{k}^{\tilde{\rho}-n}=s_{2}^{\rho_{2}} s_{3}^{\rho_{3}} \ldots s_{n}^{\rho_{n}} \beta_{n}(s)
$$

when $n=1,2, \ldots, \tilde{\rho}-1$ and $\bar{\beta}_{\tilde{\rho}}^{-}(s)=2 s_{2}^{\rho_{2}} s_{3}^{\rho_{3}} \ldots s_{n}^{\rho_{n}}$ so that $\Delta_{\tilde{\rho}}=0$, where

$$
\Delta_{\tilde{\rho}}=\left|\begin{array}{cccc}
2 & \beta_{1}(s) & \ldots & \beta_{\rho}(s) \\
\bar{\beta}_{1}(s) & 2 & \ldots & \bar{\beta}_{\rho-1}(s) \\
\vdots & \vdots & & \vdots \\
\bar{\beta}_{\bar{\rho}}(s) & \bar{\beta}_{\rho-1}(s) & \ldots & 2
\end{array}\right|=0 .
$$


Since $\operatorname{Re} f>0$ and the above Carathéodory-Toeplitz determinant $\Delta_{\rho}$ is zero we have that

$$
x_{k}(s)=\tilde{\rho}^{-1} \operatorname{Re} p\left(\varepsilon_{k}, s_{2} \varepsilon_{k}, \ldots, s_{n} \varepsilon_{k}\right) \geqslant 0, \quad k=0,1, \ldots, \tilde{\rho}-1
$$

If we set $s_{2}=z_{2}^{\tilde{\rho}}, s_{3}=z_{3}^{\tilde{\rho}}, \ldots, s_{n}=z_{n}^{\tilde{\rho}}, \varepsilon_{0}=z_{2}^{-\rho_{2}} z_{3}^{-\rho_{3}} \ldots z_{n}^{-\rho_{n}}=z^{-\rho^{*}}$ where $z \in(\partial U)^{n-1}$ we obtain the result.

(ii) $\rightarrow$ (i).

Let $f(z)=\left[p(z)+z^{\rho} \bar{p}(1 / \bar{z})\right]\left(1-z^{\rho}\right)^{-1}$ and $p \in H S_{\rho}$.

If we prove that $x_{k}(s) \geqslant 0, k=0,1, \ldots, \tilde{\rho}-1$, we, essentially, have the desired conclusion, since we can apply the maximum principle for harmonic functions, for each variable separately, thus obtaining that $f \in \Omega_{\rho}(0)$.

We consider the system

$$
\begin{gathered}
-\left(\rho_{2} \theta_{2}+\cdots+\rho_{n} \theta_{n}\right)=2 k \pi \tilde{\rho}^{-1}-2 \pi \omega_{1}, \\
\tilde{\rho} \theta_{\lambda}-\left(\rho_{2} \theta_{2}+\rho_{3} \theta_{3}+\cdots+\rho_{n} \theta_{n}\right)=2 k \pi \tilde{\rho}^{-1}-2 \pi \omega_{\lambda}, \quad \lambda=2,3 \ldots n
\end{gathered}
$$

and $k, \omega_{1}, \omega_{2}, \ldots, \omega_{n}$ integers.

The above system has a unique solution if $k=\sum_{\lambda=1}^{n} \rho_{\lambda} \omega_{\lambda}$. Since $\rho_{1}, \rho_{2}, \ldots \rho_{n}$ are numbers prime to each other, the above can be obtained by choosing suitable $\omega_{\lambda}$.

Now, if we set $s_{2}=z_{2}^{\tilde{\rho}}, s_{3}=z_{3}^{\tilde{\rho}}, \ldots, s_{n}=z_{n}^{\tilde{\rho}}, \varepsilon_{0}=z_{2}^{-\rho_{1}} z_{3}^{-\rho_{3}} \ldots \bar{z}_{n}^{\rho_{n}}=z^{-\rho^{*}}$, then $x_{0}(s) \geqslant 0$ since $p \in Q S_{\rho}$. If we replace each variable $z_{\lambda}$ by $z_{\lambda} e^{i \theta_{\lambda}}$, where $\theta_{\lambda}$ are the solutions of the previous system, we have $\operatorname{Re} p\left(u_{1}, u_{2}, \ldots, u_{n}\right) \geqslant 0$ where

$$
\begin{aligned}
& u_{1}=\varepsilon_{0} e^{-i\left(\rho_{2} \theta_{2}+\rho_{3} \theta_{3}+\cdots+\rho_{n} \theta_{n}\right)}=\varepsilon_{0} e^{2 \pi i k \tilde{\rho}^{-1}}=\varepsilon_{k} \text { and } \\
& u_{m}=z_{m}^{\tilde{\rho}} z^{-\rho^{*}} e^{i \tilde{\rho} \theta_{m}-\left(\rho_{2} \theta_{2}+\rho_{3} \theta_{3}+\ldots \rho_{n} \theta_{n}\right)}=s_{m} \varepsilon_{k}
\end{aligned}
$$

with $k=1,2, \ldots \tilde{\rho}-1$ and $m=2,3, \ldots, n$.

REMARKS 3.2.

1. If $a=\left(\rho_{1}, \rho_{2}, \ldots, \rho_{n}, 0,0, \ldots, 0\right) \in \mathbb{N}_{0}^{m}$ and $\rho=\left(\rho_{1}, \rho_{2}, \ldots, \rho_{n}\right)$, then $\Omega_{\rho}(0)=$ $\Omega_{\mathrm{a}}(0)$.

2. If $\rho \in \mathbb{N}_{0}^{n}$ with $\rho_{1}=1$ and $S_{\rho}=\left\{n \in \mathbb{N}_{0}^{n}, \theta \leqslant n \leqslant \rho, n \neq \rho, n_{1}=0\right\}, S\left(\rho^{*}\right)=$ $\left\{k \in \mathbb{N}_{0}^{n-1}, k \leqslant \rho^{*}\right\}$ then it is obvious that $H S_{\rho}$ is identical to $H S\left(\rho^{*}\right)$. Moreover, if $\left(\omega_{2}, \omega_{3}, \ldots, \omega_{n}\right) \in(\partial U)^{n-1}$ it is easy to find a $z=\left(z_{2}, z_{3}, \ldots, z_{n}\right) \in(\partial U)^{n-1}$ such that $\omega_{\lambda}=z^{-\rho^{*}} z_{\lambda}^{\tilde{\rho}}, \lambda=2,3, \ldots, n$. Hence, for every $p \in Q S_{p}$ the relation $\operatorname{Re} p\left(\varepsilon_{0}, \omega_{2}, \omega_{3}, \ldots, \omega_{n}\right) \geqslant 0$ holds. Since $n_{1}=0$, it is $\operatorname{Re} p\left(\omega_{1}, \omega_{2}, \ldots, \omega_{n}\right) \geqslant 0$ for every $\left(\omega_{1}, \omega_{2}, \ldots, \omega_{n}\right) \in(\partial U)^{n}$. Applying the maximum principle theorem for harmonic functions we obtain that $P S\left(\rho^{*}\right)=P S_{\rho}=Q S_{\rho}$. 
By means of this equality we conclude that the determination of the extreme elements of class $\Omega_{\rho}(0) \cap E P_{n}$ reduces to locating those of the class of polynomials $\operatorname{EPS}\left(\rho^{*}\right)$.

Especially in the case $\rho=(n, 1)$ the problem reduces to locating the extreme elements of the class of polynomials of degree at most $n$, which belong to the class $P_{1}$. 3. In the case of two variables the problem of locating the extreme elements of the class $\Omega_{\rho}(0) \cap E P_{2}$ is always reduced to finding the extreme elements of a class of polynomials with one variable. Indeed, if

$$
\begin{aligned}
& S_{\rho}=\left\{n \in N_{0}^{2}: n_{1} \rho_{2}-n_{2} \rho_{1}<0, n \leqslant \rho\right\} \cup\{\theta\} \quad \text { and } \\
& S_{1}=\left\{n_{2} \rho_{1}-n_{1} \rho_{2}:\left(n_{1} n_{2}\right) \in S_{\rho}\right\}
\end{aligned}
$$

we observe that the map $x\left(n_{1} n_{2}\right)=n_{2} \rho_{1}-n_{1} \rho_{2}$ is one-to-one from the set $S_{\rho}$ onto $S_{1}$, since the numbers $\rho_{1}, \rho_{2}$ are prime to each other.

If $p(z)=\sum_{n \in S_{\rho}} a_{n} z^{n}$ and define $\mathcal{L}(p)=\sum_{n \in S_{p}} a_{n} z^{n_{2} \rho_{1}-n_{1} \rho_{2}}$ then the transformation $\mathcal{L}$ is an isomorphism of the space $H S_{p}$ onto the space $H S_{1}$.

By means of Theorem 3.1 we obtain $\mathcal{L}\left[Q S_{\rho}\right]=P S_{1}$; hence

$$
\Omega_{\rho}(0) \cap E P_{2}=\left\{\left[\mathcal{L}^{-1}\left(p_{1}\right)(z)+z^{\rho} \mathcal{L}^{-1}\left(p_{1}\right)(1 / \bar{z})\right]\left(1-z^{\rho}\right)^{-1}: p_{1} \in E P S_{1}\right\}
$$

\section{Applications}

(a) If $s=\left(s_{1}, s_{2}, \ldots, s_{n}\right) \in(\partial U)^{n}$ and $\underset{\sim}{s}=\left(x_{1}, x_{2}, \ldots, x_{2 n}\right)=\left(\rho_{1}, \rho_{1}, \rho_{2}, \rho_{2}\right.$, $\left.\ldots, \rho_{n}, \rho_{n}\right) \in(\partial U)^{2 n}$, we denote by $A_{k}(s)$ the sum of products which are formed by considering all the permutations of the components of the vector $s$ taken $k$ at a time. Moreover we define $A_{0}(s)=1$.

If $S_{n}=\{0,1,2, \ldots, n\}$, then it is known that the class $E P S_{n}$ is formed by the elements of the class $P S_{n}$ which obey the relationship

$$
\operatorname{Re} p(z)=K(-1)^{n}\left(s_{1} s_{2} \ldots s_{n}\right)^{-1} z^{-n}\left(z-s_{1}\right)^{2} \ldots\left(z-s_{n}\right)^{2}
$$

for all $z \in(\partial U)^{n}$. The number $K>0$ is exactly determined through the relationship $p(0)=1$ connected with $s \in(\partial U)^{n}$. On the basis of the above considerations we conclude the following relationship:

$$
E P S_{n}=\left\{1+2(-1)^{n} A_{n}^{-1}(s) \sum_{k=1}^{n} A_{n-k}(s) z^{k}: s \in U^{n}\right\} \quad \text { (see [4]). }
$$

If we take into consideration Theorem 3.1, the above relationship solves the problem of locating the elements of class $\Omega_{\rho}(0) \cap E P_{2}$, where $\rho=(n, 1)$ or $\rho=(1, n)$. Moreover, it 
solves the problem of finding the elements of class $\Omega_{\rho}(\varphi) \cap E P_{2}$. These elements have the form $p\left(e^{i \varphi} z_{1}, z_{2}\right)$, where $p\left(z_{1}, z_{2}\right) \in \Omega_{\rho}(0) \cap E P_{2}$. The elements of the class $\Omega(\varphi) \cap E P_{2}$ coincide with the elements of the class $\Omega_{k}(\varphi) \cap E P_{\lambda}$, where $k=(n, 1,0,0 \ldots 0) \in N_{0}^{\lambda}$.

For $\rho=(1,1)$ we have

$$
\Omega_{\rho}(0) \cap E P_{2}=\left\{\left(1+s z_{1}+\bar{s} z_{2}+z_{1} z_{2}\right)\left(1-z_{1} z_{2}\right)^{-1}:|s|=1\right\} .
$$

The above result is a generalisation of the result $[2,3.3$, p.280] (that is, the function of the form $\left(1+s z_{1}+\bar{s} z_{2}+z_{1} z_{2}\right)\left(1-z_{1} z_{2}\right)^{-1},|s|=1$, belongs to the class $\left.E P_{2}\right)$.

(b) In the case $\rho=(1,1,1)$, by means of Theorem 3.1, the problem is reduced to locating the elements of class $E P S_{\rho}$ where

$$
S_{\rho}=\{(0,0),(1,0),(0,1),(1,1)\}
$$

If a polynomial belongs to the class $E P S_{\rho}$ then there is a $\left(\omega_{0} \varphi_{0}\right) \in[0,2 \pi]^{2}$ such that

$$
\operatorname{Re}\left\{1+\alpha e^{i \omega_{0}}+\beta e^{i \varphi_{0}}+\gamma e^{i\left(\omega_{0}+\varphi_{0}\right)}\right\}=0 .
$$

Without loss of generality we assume that $\left(\omega_{0}, \varphi_{0}\right)=(0,0)$. Now, since there is a minimum at this position, we obtain the following relationships:

$$
1+\alpha+\beta+\gamma=i A, \alpha+\gamma=B-1, \beta+\gamma=\Gamma,(A, B, \Gamma) \in \mathbb{R}^{3}
$$

The relationship $\operatorname{Re}\left\{1+\alpha z_{1}+\beta z_{2}+\gamma z_{1} z_{2}\right\}>0$ for all $\left(z_{1}, z_{2}\right) \in U^{2}$ by means of the maximum principle for harmonic functions, is equivalent to the relationship $\left|a+\gamma e^{i \varphi}\right| \leqslant 1+\operatorname{Re} \beta e^{i \varphi}, \varphi \in[0,2 \pi)$, so $\beta^{2}=B^{2}+A^{2} \leqslant 1$. After some algebraic manipulations we obtain

$$
\Delta_{1} \cos \varphi+\Delta_{2} \sin \varphi+\Delta_{3} \sin ^{2} \varphi-\Delta_{2} \cos \varphi \sin \varphi-\Delta_{1} \geqslant 0
$$

where

$$
\Delta_{1}=2\left(\Gamma^{2}+A^{2}+\Gamma+B\right), \Delta_{2}=-2 A B, \Delta_{3}=A^{2}-B^{2} \text {. }
$$

Now, by setting $x=\tan (\varphi / 2)(-\infty<x<\infty)$ we take $\Delta_{1}^{2}-2 \Delta_{1} \Delta_{3}-4 \Delta_{2}^{2} \geqslant 0, \Delta_{1} \leqslant 0$ which are equivalent to $\Delta_{1} \leqslant 2 B^{2}$. Next, if $H=\left\{(A, B, \Gamma) \in \mathbb{R}^{3}: \Gamma^{2}+A^{2}+\Gamma+\Gamma B \leqslant\right.$ $\left.0, A^{2}+B^{2} \leqslant 1\right\}$ we prove that

$$
\operatorname{Re}\left\{1+(i A-1-\Gamma) z_{1}+(i A-B) z_{2}+(\Gamma+B-i A) z_{1} z_{2}\right\}>0
$$

for all $(A, B, \Gamma) \in H$ and $\left(z_{1}, z_{2}\right) \in U^{2}$.

Now using a direct reversal process we can prove the converse of the previous result.

Obviously for the extreme elements which have the above form, the following holds: $f(A, B, \Gamma) \equiv \Gamma^{2}+A^{2}+\Gamma+\Gamma B=0$. If $(A, B, \Gamma) \in H$ with $f(A, B, \Gamma)=0$ and 
$s=\left(s_{1}, s_{2}, s_{3}\right) \neq(0,0,0)$ the polynomial $\tau(\lambda)=f\left(A+\lambda s_{1}, B+\lambda s_{2}, \Gamma+\lambda s_{3}\right)$ has the form $\lambda\left[\lambda\left(s_{1}^{2}+s_{2}^{2}+s_{1} s_{2}\right)+k\right], k \in \mathbb{R}$.

By the fact that $\tau(\lambda)$ assumes nonpositive values which are all to the right (or all to the left) of zero we obtain the converse result. Finally, the set $E P S_{\rho}$ is composed of polynomials of the form

$$
p(z)=1+(i A-1-\Gamma) e^{i \omega} z_{1}+(i A-B) e^{i \varphi} z_{2}+(\Gamma+B-i A) e^{i(\omega+\varphi)} z_{1} z_{2}
$$

where $(\omega, \varphi) \in[0,2 \pi)^{2},(A, B, \Gamma) \in \mathbb{R}^{3}, \Gamma^{2}+A^{2}+\Gamma+\Gamma B=0, A^{2}+B^{2} \leqslant 1$.

\section{REFERENCES}

[1] F. Forelli, 'A necessary condition on the extreme points of a class of holomorphic functions', Pacific J. Math. 73 (1977), 81-86.

[2] F. Forelli, 'A necessary condition on the extreme points of a class of holomorphic functions II', Pacific J. Math. 92 (1981), 277-281.

[3] F. Holland, 'The extreme points of a class of functions with positive real part', Math. Ann. 202 (1973), 85-87.

[4] J.N. McDonald, 'Convex sets of operators on the disk algebra', Duke Math. J. 42 (1975), 787-796.

Department of Mathematics

Faculty of Sciences

University of Patras

26110 Patras

Greece 